\title{
Application of Cre/LoxP system for induction of multiple chromosomal aberrations in the human genome
}

\author{
Mungalov R. ${ }^{1,2 *}$, Khabarova A. ${ }^{1}$, Fishman V. ${ }^{1}$ \\ ${ }^{1}$ Institute of Cytology and Genetics, SB RAS, Novosibirsk, Russia \\ ${ }^{2}$ Novosibirsk State University, Novosibirsk, Russia \\ *e-mail:mungalov.roman@yandex.ru
}

Key words: Cre/LoxP system, genome architecture, gene expression

Motivation and Aim: In this research we have a goal to study an interplay between disturbances of three-dimensional human genome organization [1], induced by chromosomal aberrations, and gene expression and epigenetic modifications. We suggest an alternative way of the induced mutagenesis, namely Cre/LoxP system. It will allow us to create a big collection of chromosomal aberrations (up to 10 events per cell), in spite of unique events using CRISPR/Cas9 [2].The analysis of just a few number of subclones, each of them will have about 10 aberrations, will allow us to get enough information about gene expression changes in different parts of the human genome.

Methods and Algorithms: We use Cre/LoxP system [3] to induce chromosomal aberrations in near-haploid human cell line HAP-1. We apply lentiviruses as vectors for transporting LoxP-sites in random parts of human genome and then perform exogenic expression of Cre-recombinase, inducing different recombinations between LoxP-sites. Number of LoxP-site integrations and recombinations we estimate using qPCR. To localize LoxP-site's integrations in the genome we are planning to create inverse-PCR library of subclones, carrying different numbers of LoxP-sites. Then this library will be sequenced. Several subclones will be further analyzed by capture $\mathrm{HiC}$ and bioinformatics modeling of the genome 3D-landscape.

Results: We have obtained about 50 subclones, carrying various number of LoxP-site integrations, and sequenced ones with the largest number of integrations to localize LoxP-insertions. To improve a system of transporting LoxP-sites to the genome we have constructed new vectors, based on Sleeping Beauty transposons.

Conclusion: Using Cre/LoxP system we have achieved an integration of about 25 LoxPsites (maximum now) in the human near-haploid cell line HAP-1. In addition, we have sequenced subclones with the maximum number of LoxP-site integrations. Further recombination and modeling of 3D-landscape will show the interplay between the genome architecture and gene expression.

\section{References}

1. Rowley M.J., Corces V.G. Organizational principles of 3D genome architecture. Nat. Rev. Genet. 2018;19:1.

2. Lupiáñez D. G. et al. Disruptions of topological chromatin domains cause pathogenic rewiring of geneenhancer interactions. Cell. 2015;161(5):1012-1025.

3. Sauer B. et al. Site-specific DNA recombination in mammalian cells by the Cre recombinase of bacteriophage P1. Proc. Natl. Acad. Sci. 1988;85(14):5166-5170. 